\title{
Insect galls in Rupestrian field and Cerrado stricto sensu vegetation in Caetité, Bahia, Brazil
}

\author{
Leir Guimarães Vieira ${ }^{1}$, Ravena Malheiros Nogueira², Elaine Cotrim Costa ${ }^{3}$, \\ Sheila Patrícia Carvalho-Fernandes ${ }^{4}$ \& Juliana Santos Silva ${ }^{2 *}$ [i] \\ ${ }^{1}$ Universidade do Estado da Bahia, Departamento de Ciências Humanas, Campus VI, Caetité, BA, Brasil \\ ${ }^{2}$ Universidade do Estado da Bahia, Departamento de Educação, Campus VIII, Paulo Afonso, BA, Brasil \\ ${ }^{3}$ Universidade Federal de Minas Gerais, Departamento de Botânica, Belo Horizonte, MG, Brasil \\ ${ }^{4}$ Universidade Federal do Rio de Janeiro, Museu Nacional, Departamento de Entomologia, Quinta da Boa \\ Vista, São Cristóvão, RJ, Brasil \\ *Corresponding author: Juliana Santos Silva, e-mail: jussilva@uneb.br
}

VIEIRA, L. G.; NOGUEIRA, R. M.; COSTA, E. C.; CARVALHO-FERNANDES, S. P.; SANTOS-SILVA, J. Insect galls in Rupestrian field and Cerrado stricto sensu vegetation in Caetité, Bahia, Brazil. Biota Neotropica. 18(2): e20170402. http://dx.doi.org/10.1590/1676-0611-BN-2017-0402

\begin{abstract}
We investigated insect galls in Rupestrian field and Cerrado vegetation in the municipality of Caetité (BA), Brazil, between August/2015 and June/2016. This is the first study of gall diversity in Rupestrian field vegetation in that state. We encountered 48 different morphotypes of galls, distributed among 17 different plant species belonging to 13 genera and 21 host plant families. The greatest gall richness was observed in Cerrado $(n=39)$ as compared to Rupestrian field $(n=9)$ vegetation. The principal botanical families observed with galls were Leguminosae, Malpighiaceae, and Myrtaceae, with 10, 8 and 4 morphotypes, respectively. The genera showing the greatest gall diversity were Copaifera L. ( $\mathrm{n}=6)$ (Leguminosae-Detarioideae), Croton L. (n=2) (Euphorbiaceae), Mimosa L. (n=2) (Leguminosae-Caesalpinioideae), Byrsonima Rich. ex Kunth ( $\mathrm{n}=2)$ (Malpighiaceae), and Eugenia L. (n=2) (Myrtaceae). The super-host species was Copaifera sabulicola J.A.S. Costa \& L.P. Queiroz ( $\mathrm{n}=$ 5). Most galls occurred on leaves, being globoid, glabrous, isolated, and unilocular. Most gall-inducing insect species belong to the Cecidomyiidae family, while the associated fauna was represented by Hymenoptera and Lepidoptera. We recorded here for the first time galls on Ocotea velutina (Mart. ex Ness) Rohwer (Lauraceae) and Miconia alborufescens Naudin (Melastomataceae). The results indicate the need for more studies to understand the dynamics of the insect-induced galls in Rupestrian field.
\end{abstract}

Keywords: Gall-inducing insects, plant-insect interactions, northeastern Brazil, semiarid region.

\section{Galhas de insetos em vegetação de Campo Rupestre e de Cerrado stricto sensu de Caetité, Bahia, Brasil}

\footnotetext{
Resumo: Este estudo investigou e caracterizou as galhas de uma área de Campo rupestre e de Cerrado no município de Caetité (BA) no período de agosto de 2015 a junho de 2016. Esta é a primeira contribuição para o conhecimento da diversidade de galhas de Campo rupestre neste estado. Foram registrados 48 morfotipos de galhas distribuídas em 17 espécies distintas, pertencentes a 13 gêneros e 21 famílias de plantas hospedeiras. A maior riqueza de galhas foi observada no Cerrado $(n=39)$, quando comparada com o Campo rupestre $(n=9)$. As famílias botânicas mais ricas em galhas foram Leguminosae, Malpighiaceae e Myrtaceae, com 10, 8 e 4 morfotipos, respectivamente. Os gêneros com maior diversidade de galhas foram Copaifera L. ( $\mathrm{n}=6$ ) (Leguminosae-Detarioideae), Croton L. ( $\mathrm{n}=2)$ (Euphorbiaceae), Mimosa L. (n=2) (Leguminosae-Caesalpinioideae), Byrsonima Rich. ex Kunth (n=2) (Malpighiaceae) e Eugenia L. (n=2) (Myrtaceae). A espécie super-hospedeira foi Copaifera sabulicola J.A.S. Costa \& L.P. Queiroz $(n=5)$. A maioria das galhas ocorreu nas folhas, sendo globoides, glabras, isoladas e uniloculares. A maioria dos indutores pertence à família Cecidomyiidae, enquanto que a fauna associada está representada por Hymenoptera e Lepidoptera. Foram registradas pela primeira vez galhas em Ocotea velutina (Mart. ex Ness) Rohwer (Lauraceae) e Miconia alborufescens Naudin (Melastomataceae). Os resultados encontrados indicam a necessidade de mais estudos para entender a dinâmica dos insetos indutores de galhas em Campos rupestres.

Palavras-chave: Insetos galhadores, interação inseto-planta, Nordeste brasileiro, semiárido.
} 


\section{Introduction}

Galls are highly specialized structures resulting from the atypical development of plant tissues induced by the presence and activities of nematodes, bacteria, fungi, insects, mites and other plants (Mani, 1964). Insect galls are one of the most sophisticated forms of insectplant interactions, with the galls providing adaptive advantages to the gall-inducing insects through the provision of food resources and physical protection against natural enemies and environmental stress (Mani 1964, Price et al. 1987, Stone \& Schönrogge 2003). The capacity to induce galls in plants evolved numerous times between and within insect orders, with current representatives of gall-inducing species among the Diptera, Hymenoptera, Hemiptera, Coleoptera, Lepidoptera, and Thysanoptera (Mani 1964, Espírito-Santo \& Fernandes 2007). It is estimated that there are approximately 21,000 to 211,000 species of gall-inducing insects in the world (Espírito-Santo \& Fernandes 2007).

The known diversity of galls in Brazil is largely based on studies undertaken in Cerrado, Rupestrian field, Cerradão, and dry forest environments in the southeastern region of the country (e.g., Gonçalves-Alvim \& Fernandes 2001, Maia \& Fernandes 2004, Carneiro et al. 2009, Coelho et al. 2009, 2013a). In spite of recent progress in studies of gall-inducing insects and their host plants in those ecosystems, there is still much to be discovered concerning their patterns of distribution and diversity, especially in the northeastern region of Brazil. Only four inventories of galls in Cerrado phytophysiognomies (Nogueira et al., 2016, Costa 2016) and in Caatinga-Cerrado transition areas (Costa et al. 2014a, b) in Bahia State, for example, have so far been undertaken.

The municipality of Caetité, located in southeastern Bahia State, presents a diverse landscape in terms of both geological and vegetational aspects. The higher elevation and plains areas of the municipality have phytophysiognomies typical of the Cerrado (neotropical savanna). Small areas of Rupestrian field vegetation, growing on quartzite rock exposures, are also found in Caetité. Arboreal and shrub Caatinga dryland vegetational formations are principally encountered on mountainsides; Gallery forests are essentially restricted to water courses and constitute the most humid environments (Vasconcelos et al. 2012).

This environmental diversity is reflected in high regional biodiversity, and presents significant challenges to ecological and taxonomic studies - and specially to conservation efforts. The flora and fauna of Caetite are still poorly known, although the region is considered to be of high conservation priority (MMA/Portaria ${ }^{\circ} 9$ de 23/01/2007). The conservation of the biodiversity of Caetite is threatened by deforestation, burning, mechanized and irrigated agriculture, mining, and the installation of wind-farms. The exploitation of natural resources in the municipality of Caetite has resulted in the loss and fragmentation of natural habitats, thereby diminishing biodiversity and interfering with natural interactions between insects and plants (Tabarelli, 1998). As such, it is increasingly important to examine gall-inducing insects and their host plants, as they can contribute to conservation strategies as indicators of environmental quality.

Within that context, we inventoried an area of Cerrado and Rupestrian field vegetation in the municipality of Caetité to characterize gall morphologies and identify host plants and gall-inducing insects. This study represents the first examination of gall diversity in an area of Rupestrian field vegetation in Bahia State.

\section{Materials and Methods}

Current study was carried out in Brejinho das Ametistas, a district within the municipality of Caetité, in central-southern Bahia

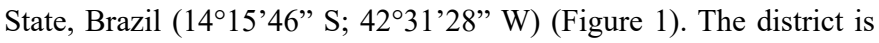
situated on a high plain with elevations of 750 to $1.100 \mathrm{~m}$ above sea level, approximately $20 \mathrm{~km}$ from the town of Caetité (CEI 1994). The vegetation is quite complex and heterogeneous, with areas of Caatinga, Rupestrian field, Cerrado, and Gallery forest vegetation, influenced by soil types, and the elevation and inclination of the land. Phytophysiognomies typical of Cerrado (locally known as "geraes") occur on plains at elevations above $750 \mathrm{~m}$. Small areas of Rupestrian field vegetation are encountered on quartzite rock outcrops at elevations at or above $1000 \mathrm{~m}$ (Vasconcelos et al. 2012).

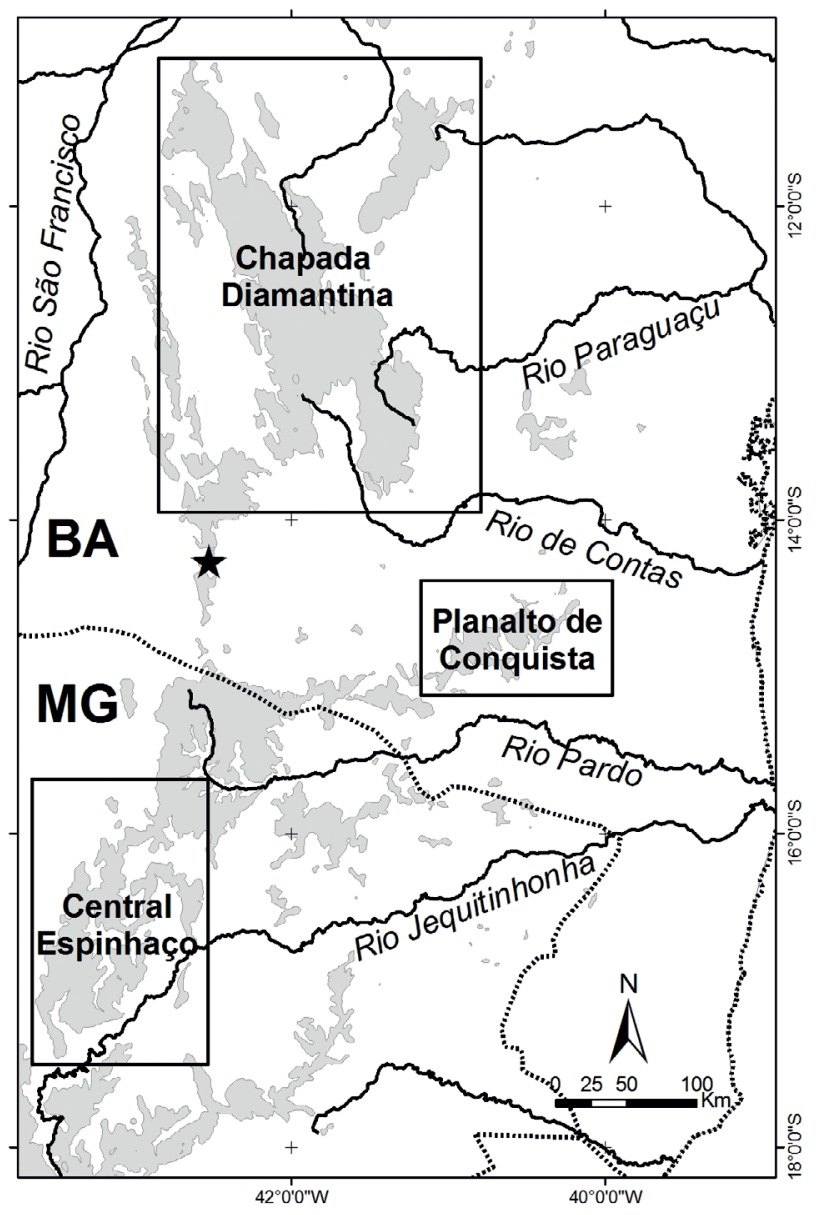

Figure 1. Location of the district of Brejinho das Ametistas, Caetité, Bahia State, Brazil. Brazilian states: $\mathrm{BA}=$ Bahia; $\mathrm{MG}=$ Minas Gerais. Source: Vasconcelos et al. (2012)

Ten surveys were undertaken at monthly intervals between August/2015 and June/2016 in areas of Rupestrian field and Cerrado vegetation. Collections of galls and their host plants were made in each of those areas for four hours during each visit, totaling 40 hours of collection efforts by two to three people. The area was searched for galls, which were photographed, collected, and stored in plastic bags for removal to the laboratory. The morphological characteristics of the galls 
were noted, including their colors, occurrence on plant organs, pilosity, isolated/grouped, and shapes, following the terminology proposed by Isaias et al. (2013).

The host plants were collected, pressed, and deposited in the Caetité Collection of the HUNEB herbarium. Some of the galls were stored in plastic containers in the laboratory together with moistened paper towels to maintain humidity levels and allow the emergence of the inducing insects (or any associated fauna). Other samples were dissected under a stereomicroscope to extract larva and to determine the number of chambers in each gall. Both the larva and the emerging winged insects were preserved in $70 \%$ ethanol. The identifications of the insect species (when adult insects did not emerge) was undertaken by comparisons with the morphotypes of known gall-inducing insects and host plants previously identified in Cerrado and Rupestrian field environments in Brazil (e.g, Carneiro et al. 2009, Araújo et al., 2012, Nogueira et al. 2016). All the examined material was deposited in the Entomological Collection of the Museu Nacional (MN/UFRJ).

The identifications of the host plants were principally made through the use of analytical keys provided in classic publications, regional floras, and taxonomic revisions, as well as by comparisons with specimens previously identified by specialists and deposited in the HUNEB and HUEFS herbaria. The taxa are presented here in alphabetical order by family, following APG IV (2016).

\section{Results}

We collected 48 different morphotypes of galls distributed on 37 distinct species of host plants belonging to 25 genera and 21 families (Table 1; Figures 2-4). The greatest richness of galls was encountered in Cerrado $(n=39 ; 81 \%)$ as compared to Rupestrian field vegetation $(n=9$; $19 \%)$. The mean number of gall morphotypes seen on a single host plant was 1.3. The botanical families that hosted the greatest richness of gall morphologies were Leguminosae, Malpighiaceae, and Myrtaceae (with 10, 8 and 4 respectively); those same families demonstrated the greatest numbers of host species (5, 7 and 4 galls respectively) (Table 1). The plant genera with the greatest richness of galls were Copaifera Desf. ( $\mathrm{n}=6)$ (Leguminosae-Detarioideae), Croton L. $(\mathrm{n}=2)$ (Euphorbiaceae), Mimosa L. (n=2) (Leguminosae-Caesalpinioideae), Byrsonima Rich. ex Kunth (n=2) (Malpighiaceae), and Eugenia L. $(n=2)$ (Myrtaceae). The super-host species demonstrating the greatest diversity of gall morphotypes was Copaifera sabulicola J.A.S. Costa \& L.P. Queiroz ( $\mathrm{n}=5$ morphotypes). Galls were recorded for the first time on Ocotea velutina (Mart. ex Ness) Rohwer (Lauraceae) and Miconia alborufescens Naudin (Melastomataceae) (Figures 2M; 3K).

More than $80 \%$ of the gall morphotypes were induced on leaves, and were distributed all over their surfaces. The stems were the second host organ in terms of frequency (15\%). Stems galls were observed on Guapira opposita (Vell.) Reitz, Eugenia punicifolia (Kunth) DC., Byrsonima cf. guilleminiana A. Juss., and C. sabulicola, most being fusiform or irregular and located near the internodes. Most galls were found on only one host plant organ (81\%), with the exception of Guapira opposita and Byrsonima cf. guilleminiana (which had galls on both their stems and leaves) and C. sabulicola (which hosted galls on buds, leaves, and stems).
Most of the gall morphotypes were of the same color of the host plant organ on which they occurred, with green galls predominating on leaves (54\%) and brown galls being predominantly observed on stems (15\%). Galls were classified into five different basic shapes: globoid (67\%), lenticular (16\%), conical (6\%), marginal roll $(6 \%)$, and cylindrical (2\%). We also observed amorphous galls in $2 \%$ of the specimens analyzed. Galls can occur isolated or grouped on host plants. Currently, the isolated form was predominant (69\% of all galls). In terms of the number of chambers, unilocular galls were predominant, been observed in $73 \%$ of the morphotypes, only $27 \%$ of the galls were multilocular. In relation to the pilosity, $33 \%$ of the morphotypes have trichomes, while $67 \%$ were glabrous.

Of the 48 galls observed, we were able to identify the inducing insects only in 17, all of which belong to the Cecidomyiidae (Diptera). We encountered considerable difficulty in precisely identifying the insects principally due to the absence of any inducing insect species and the high frequency of galls containing only their immature phases. The fauna associated with those galls were Hymenoptera and Lepidoptera as parasitoids and inquilines, respectively.

\section{Discussion}

The richness of insect galls on Brejinho das Ametistas is greater than that of the other Rupestrian field or Cerrado areas investigated in Brazil (Table 2); the mean of 1.3 gall morphotypes per host plant species was only slightly less than the mean values recorded in other studies, such as that in the Pirapitinga Ecological Station - MG (with 1.5 morphotypes; Gonçalves-Alvim \& Fernandes 2001), Caldas Novas - GO (1.6 morphotypes; Santos et al. 2012), Cadeia do Espinhaço - MG (1.7 morphotypes; Carneiro et al. 2009), and Serra do Cipó - MG (1.8 morphotypes; Coelho et al. 2009).

In surveys of entomogenous galls undertaken in different regions of Brazil, the Leguminosae, Malpighiaceae, and Myrtaceae stood out as hosting a rich diversity of gall-inducing insects, with many species bearing galls (e.g., Santos et al. 2009, 2011, Coelho et al. 2009, Silva et al. 2011, Carvalho-Fernandes et al. 2012, Luz et al. 2012, Santos et al. 2012, Maia, 2013a,b, Maia \& Fernandes, 2004, Costa et al. 2014a, Nogueira et al. 2016). Among the families mentioned in this inventory, Leguminosae has been frequent as the main host in different phytophysiognomies in Brazil, such as Rupestrian field (Carneiro et al. 2009), Amazon rainforest (Almada \& Fernandes 2011), and Semideciduous Seasonal Forest (Santos \& Ribeiro 2015). Those plant families comprise high numbers of species and are amply distributed throughout the Neotropical region, which favors their association with gall-inducing insects (Maia 2001)

The genus demonstrating the greatest richness of galls was Copaifera, differing from observations made in other regions of Brazil. A compilation of studies of galls undertaken in Goiás State (in the central-western region of Brazil) showed Myrcia DC. (Myrtaceae) and Qualea Aubl. (Vochysiaceae) to be the principal super-host genera of galls in Cerrado sensu stricto areas (Araújo et al. 2014), and Ocotea Aubl. (Lauraceae) were the most diverse host plant taxa (Araújo et al. 2012) in areas of Floresta Ombrófila Densa. 
Vieira, L.G. et al.

Table 1. Characterization of insect galls from Rupestrian field and Cerrado stricto sensu vegetation in Caetité, BA, Brazil. + recorded for the first time, * provided in classical publications and $* *$ material obtained

\begin{tabular}{|c|c|c|c|c|c|c|c|c|c|c|c|}
\hline Host plant Family/Species & Organ & Side & Color & Shape & Pubescense & Group & Chambers & Gall maker & $\begin{array}{l}\text { Associated } \\
\text { fauna }\end{array}$ & Figures & Vegetation \\
\hline \multicolumn{12}{|l|}{ ANNONACEAE } \\
\hline Annona sp. & Leaf & Adaxial & Green & Globoid & Yes & Isolated & 1 & Not identified & Not identified & $2 \mathrm{~A}$ & Cerrado \\
\hline $\begin{array}{l}\text { Duguetia furfuracea } \\
\text { (A.St.-Hil.) Saff. }\end{array}$ & Leaf & Adaxial & Green & Globoid & Yes & Grouped & 1 & $\begin{array}{l}\text { Bruggmanniella } \\
\text { sp.* }\end{array}$ & Not identified & 2B & Cerrado \\
\hline \multicolumn{12}{|l|}{ ANACARDIACEAE } \\
\hline Anacardium humile A.St.-Hil. & Leaf & $\begin{array}{r}\text { Adaxial/ } \\
\text { Abaxial }\end{array}$ & Green & Globoid & No & Grouped & 1 & Cecidomyiidae** & Not identified & $2 \mathrm{C}$ & Cerrado \\
\hline \multicolumn{12}{|l|}{ BIGNONIACEAE } \\
\hline Bignoniaceae Indet. & Stem & Abaxial & Brown & Globoid & No & Isolated & Various & Not identified & Not identified & $2 \mathrm{D}$ & $\begin{array}{l}\text { Rupestrian } \\
\text { field }\end{array}$ \\
\hline \multicolumn{12}{|l|}{ CONVOVULACEAE } \\
\hline Ipomoea sp. & Leaf & Adaxial & Yellow & Globoid & Yes & Isolated & 1 & Not identified & Not identified & $2 \mathrm{E}$ & $\begin{array}{l}\text { Rupestrian } \\
\quad \text { field }\end{array}$ \\
\hline \multicolumn{12}{|l|}{ COMBRETACEAE } \\
\hline Combretum leprosum Mart. & Leaf & $\begin{array}{r}\text { Adaxial/ } \\
\text { Abaxial }\end{array}$ & $\begin{array}{l}\text { Green/ } \\
\text { Yellow }\end{array}$ & Globoid & No & Grouped & 1 & Cecidomyiidae** & Lepidoptera** & $2 \mathrm{~F}$ & Cerrado \\
\hline $\begin{array}{l}\text { Terminalia fagifolia Mart. } \\
\text { DILLENIACEAE }\end{array}$ & Leaf & Abaxial & Brown & Conical & Yes & Isolated & 1 & Not identified & Not identified & $2 \mathrm{G}$ & Cerrado \\
\hline Davilla rugosa Poir. & Fruit & Adaxial & Brown & Globoid & No & Grouped & 1 & Not identified & Not identified & $2 \mathrm{H}$ & Cerrado \\
\hline \multicolumn{12}{|l|}{ EBENACEAE } \\
\hline Dyosprus sp. & Leaf & Adaxial & Green & Lenticular & No & Grouped & 1 & Not identified & Not identified & $2 \mathrm{I}$ & Cerrado \\
\hline \multicolumn{12}{|l|}{ EUPHORBIACEAE } \\
\hline Croton sp. 1 & Leaf & Adaxial & Green & Cylindrical & Yes & Isolated & 1 & Not identified & Hymenoptera** & $2 \mathrm{~J}$ & Cerrado \\
\hline Croton sp. 2 & Leaf & Abaxial & Brown & Globoid & No & Grouped & Various & Not identified & Not identified & $2 \mathrm{~L}$ & Cerrado \\
\hline $\begin{array}{l}\text { LAURACEAE } \\
+ \text { Ocotea velutina } \\
\text { (Mart. ex Ness) Rohwer }\end{array}$ & Leaf & $\begin{array}{l}\text { Adaxial/ } \\
\text { Abaxial }\end{array}$ & $\begin{array}{l}\text { Green/ } \\
\text { Brown }\end{array}$ & Globoid & Yes & Grouped & 1 & Not identified & Not identified & $2 \mathrm{M}$ & Cerrado \\
\hline \multicolumn{12}{|l|}{ LEGUMINOSAE } \\
\hline Leguminosae Indet. 1 & Leaf & Adaxial & Brown & Globoid & No & Isolated & 1 & Cecidomyiidae** & Not identified & $2 \mathrm{~N}$ & Cerrado \\
\hline Leguminosae Indet. 2 & Leaf & Adaxial & Green & $\begin{array}{l}\text { Marginal } \\
\text { roll }\end{array}$ & No & Isolated & 1 & Cecidomyiidae** & Not identified & $2 \mathrm{O}$ & $\begin{array}{l}\text { Rupestrian } \\
\quad \text { field }\end{array}$ \\
\hline \multicolumn{12}{|l|}{$\begin{array}{l}\text { LEGUMINOSAE- } \\
\text { CAESALPINIOIDEAE }\end{array}$} \\
\hline \multirow[t]{2}{*}{ Mimosa gemmulata Barneby } & Leaf & Adaxial & Green & $\begin{array}{l}\text { Marginal } \\
\text { roll }\end{array}$ & No & Isolated & 1 & Cecidomyiidae** & Not identified & $2 \mathrm{P}$ & Cerrado \\
\hline & Leaf & Adaxial & Red & Globoid & Yes & Isolated & 1 & Cecidomyiidae** & Not identified & $2 \mathrm{Q}$ & Cerrado \\
\hline \multicolumn{12}{|l|}{$\begin{array}{l}\text { LEGUMINOSAE- } \\
\text { DETARIOIDEAE }\end{array}$} \\
\hline Copaifera langsdorffii Desf. & Leaf & $\begin{array}{c}\text { Adaxial/ } \\
\text { Abaxial }\end{array}$ & Green & Lenticular & No & Grouped & 1 & Cecidomyiidae** & Not identified & $3 \mathrm{~A}$ & Cerrado \\
\hline \multirow{5}{*}{$\begin{array}{l}\text { Copaifera sabulicola J.A.S. } \\
\text { Costa \& L.P. Queiroz }\end{array}$} & Leaf & Adaxial & Brown & Globoid & Yes & Isolated & 1 & Not identified & Not identified & $3 \mathrm{~B}$ & Cerrado \\
\hline & Stem & Abaxial & Brown & Globoid & No & Grouped & Various & Not identified & Not identified & $3 \mathrm{C}$ & Cerrado \\
\hline & Bud & Abaxial & Brown & Amorphous & No & Isolated & 1 & Not identified & Not identified & $3 \mathrm{D}$ & Cerrado \\
\hline & Leaf & Adaxial & Green & Globoid & Yes & Isolated & 1 & Not identified & Not identified & $3 \mathrm{E}$ & Cerrado \\
\hline & Stem & Abaxial & Brown & Globoid & No & Isolated & Various & Not identified & Not identified & $3 \mathrm{~F}$ & Cerrado \\
\hline \multicolumn{12}{|l|}{ MALPIGHIACEAE } \\
\hline \multirow{2}{*}{$\begin{array}{l}\text { Byrsonima cf. guilleminiana } \\
\text { A. Juss. }\end{array}$} & Leaf & Abaxial & Green & Conical & Yes & Isolated & 1 & Cecidomyiidae* & Not identified & $3 \mathrm{G}$ & Cerrado \\
\hline & Stem & - & Brown & Fusiform & No & Isolated & Various & Not identified & Not identified & $3 \mathrm{H}$ & Cerrado \\
\hline Malphighiaceae Indet.1 & Stem & - & Brown & Globoid & Yes & Isolated & 1 & Not identified & Hymenoptera $^{* *}$ & $3 \mathrm{I}$ & Cerrado \\
\hline Malphighiaceae Indet. 2 & Leaf & Adaxial & White & Globoid & Yes & Grouped & 1 & Not identified & Not identified & $3 \mathrm{~J}$ & Cerrado \\
\hline
\end{tabular}


Continued Table 1.

\begin{tabular}{|c|c|c|c|c|c|c|c|c|c|c|c|}
\hline Host plant Family/Species & Organ & Side & Color & Shape & Pubescense & Group & Chambers & Gall maker & $\begin{array}{l}\text { Associated } \\
\text { fauna }\end{array}$ & Figures & Vegetation \\
\hline Malphighiaceae Indet. 3 & Leaf & $\begin{array}{c}\text { Adaxial/ } \\
\text { Abaxial }\end{array}$ & Brown & Globoid & Yes & Isolated & Various & Cecidomyiidae** & Hymenoptera** & $3 \mathrm{~L}$ & Cerrado \\
\hline Malphighiaceae Indet. 4 & Leaf & Abaxial & White & Globoid & Yes & Isolated & 1 & Not identified & Hymenoptera** & $3 \mathrm{M}$ & Cerrado \\
\hline Malphighiaceae Indet. 5 & Leaf & Adaxial & Green & Globoid & Yes & Isolated & 1 & Not identified & Not identified & $3 \mathrm{~N}$ & Cerrado \\
\hline Malphighiaceae Indet. 6 & Leaf & Adaxial & Green & Globoid & No & Isolated & 1 & Not identified & Not identified & 30 & Cerrado \\
\hline \multicolumn{12}{|l|}{ MALVACEAE } \\
\hline \multicolumn{11}{|l|}{ MELASTOMATACEAE } & Cerrado \\
\hline $\begin{array}{l}\text { Miconia alborufescens } \\
\text { Naudin }\end{array}$ & Leaf & Adaxial & White & Globoid & Yes & Grouped & 1 & Not identified & Not identified & $3 \mathrm{Q}$ & Cerrado \\
\hline \multicolumn{12}{|l|}{ MYRTACEAE } \\
\hline Campomanesia sp. & Leaf & Adaxial & Green & Lenticular & No & Isolated & 1 & Not identified & Hymenoptera** & $4 \mathrm{~A}$ & Cerrado \\
\hline $\begin{array}{l}\text { Eugenia punicifolia (Kunth) } \\
\text { DC. }\end{array}$ & Stem & - & Brown & Globoid & No & Isolated & 1 & Not identified & Hymenoptera** & $4 \mathrm{~B}$ & Cerrado \\
\hline Eugenia sp. & Leaf & Adaxial & Green & Lenticular & No & Isolated & 1 & Not identified & Hymenoptera** & $4 \mathrm{C}$ & Cerrado \\
\hline \multicolumn{11}{|l|}{ NYCTAGINACEAE } & Cerrado \\
\hline \multirow[t]{2}{*}{ Guapira opposita (Vell.) Reitz } & Stem & Abaxial & Brown & Globoid & No & Isolated & Various & Cecidomyiidae* & Hymenoptera* & $4 \mathrm{E}$ & $\begin{array}{l}\text { Rupestrian } \\
\text { field }\end{array}$ \\
\hline & Leaf & Adaxial & Brown & Globoid & No & Isolated & 1 & Diptera* & Not identified & $4 \mathrm{~F}$ & $\begin{array}{l}\text { Rupestrian } \\
\text { field }\end{array}$ \\
\hline \multicolumn{12}{|l|}{ OCHNACEAE } \\
\hline \multirow[t]{3}{*}{ Ouratea sp. } & Leaf & Adaxial & Green & Lenticular & No & Isolated & 1 & Cecidomyiidae** & Not identified & $4 \mathrm{G}$ & Cerrado \\
\hline & Leaf & $\begin{array}{l}\text { Adaxial/ } \\
\text { Abaxial }\end{array}$ & Green & Globoid & No & Grouped & Various & Not identified & Not identified & $4 \mathrm{H}$ & Cerrado \\
\hline & Leaf & Adaxial & Green & Lenticular & No & Isolated & 1 & Not identified & Not identified & $4 \mathrm{I}$ & Cerrado \\
\hline \multicolumn{12}{|l|}{ PROTEACEAE } \\
\hline \multirow[t]{2}{*}{ Roupala montana Aubl. } & Leaf & Abaxial & Green & $\begin{array}{l}\text { Marginal } \\
\text { roll }\end{array}$ & No & Isolated & 1 & Not identified & Not identified & $4 \mathrm{~J}$ & $\begin{array}{l}\text { Rupestrian } \\
\text { field }\end{array}$ \\
\hline & Leaf & Adaxial & Green & Lenticular & No & Isolated & 1 & Not identified & Not identified & $4 \mathrm{~L}$ & $\begin{array}{l}\text { Rupestrian } \\
\text { field }\end{array}$ \\
\hline \multicolumn{12}{|l|}{ RUTACEAE } \\
\hline \multicolumn{12}{|l|}{ SAPINDACEAE } \\
\hline \multicolumn{12}{|l|}{ TRIGONEACEAE } \\
\hline \multicolumn{11}{|l|}{ VERBENACEAE } & Cerrado \\
\hline $\begin{array}{l}\text { Lippia cf. alba (Mill.) N.E. Br. } \\
\text { ex Britton \& P. Wilson }\end{array}$ & Leaf & Adaxial & Green & Globoid & Yes & Isolated & 1 & Cecidomyiidae** & Hymenoptera** & $4 \mathrm{P}$ & $\begin{array}{l}\text { Rupestrian } \\
\text { field }\end{array}$ \\
\hline Lippia microphylla Cham. & Leaf & Abaxial & $\begin{array}{l}\text { Green/ } \\
\text { Brown }\end{array}$ & Globoid & Yes & Isolated & 1 & Cecidomyiidae** & Not identified & $4 \mathrm{Q}$ & $\begin{array}{l}\text { Rupestrian } \\
\text { field }\end{array}$ \\
\hline
\end{tabular}

The richness of morphotypes of galls in Copaifera is due to the presence of the super host Copaifera langsdorffii Desf., in which more of 20 morphotypes were reported (Costa et al. 2010, Maia et al. 2013a, Nogueira et al. 2016). In this study, we reported for the first time $C$. sabulicola as a super host of galls for Cerrado areas. However, this diversity of morphotypes in Copaifera species should be investigated with phenological approaches, since, galls on C. langsdorffi $i$ vary in shape along their development (Oliveira et al., 2008, Oliveira et al., 2014).
In addition to the presence of super host species, the floristic composition of the vegetation contributes to the diversity of morphotypes of galls in different phytophysiognomies (Veldtman \& Macgeoch 2003, Fleck \& Fonseca 2007). This can be observed in this study, in which the host plants were reported in other inventories. Duguetia furfuracea (A.St.-Hil.) Saff., Terminalia fagifolia Mart, Mimosa gemmulata Barneby, Combretum leprosum Mart., Byrsonima guilleminiana A. Juss., Luehea divaricata Mart., Lippia microphylla Cham. and Trigonia nivea Cambess. were recorded in other areas of 

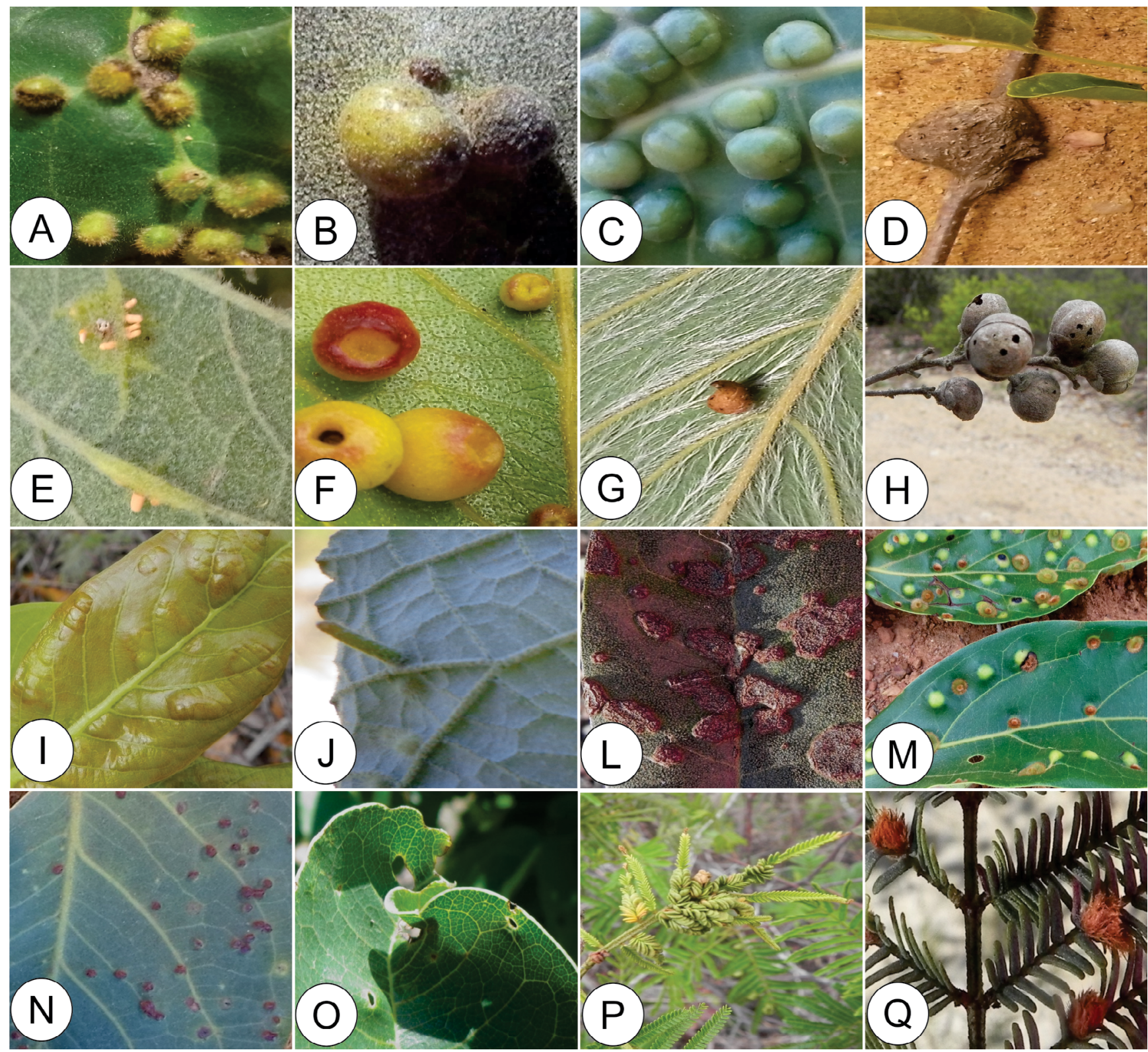

Figure 2. Insect galls in Rupestrian field and Cerrado stricto sensu vegetation in Caetité, BA, Brazil. A. Leaf gall on Annona sp. (Annonaceae). B. Leaf gall induced by Bruggmanniella sp. (Cecidomyiidae) on Duguetia furfuracea (Annonaceae). C. Leaf gall induced by Cecidomyiidae on Anacardium humile (Anacardiaceae). D. Stem gall on Bignoniaceae Indet. E. Leaf gall on Ipomea sp. (Convovulaceae). F. Leaf gall induced by Cecidomyiidae on Combretum leprosum (Combretaceae). G. Leaf gall on Terminalia fagifolia (Combretaceae). H. Fruit gall on Davilla rugosa (Dilleniaceae). I. Leaf gall on Dyosprus sp. (Ebenaceae). J. Leaf gall on Croton sp. 1 (Euphorbiaceae). L. Leaf gall on Croton sp. 2. M. Leaf gall on Ocotea velutina (Lauraceae). N. Leaf gall on Leguminosae Indet. 1. O. Marginal roll gall on Leguminosae Indet. 2 P-Q. Pinnula galls induced by Cecidomyiidae on Mimosa gemmulata (Leguminosae-Caesalpinioideae). P. Leaf gall. Q. Leaf gall.

Cerrado and/or Caatinga-Cerrado transition (Coelho et al. 2013b, Costa et al. 2014a,b, Urso-Guimarães \& Scareli-Santos 2006, Nogueira et al. 2016, Urso-Guimarães et al. 2017). Anacardium humile A.St.-Hil. and Eugenia punicifolia (Kunth) DC., besides being host plants in areas of Cerrado, were reported in Rupestrian field and Gallery forest (Araújo et al. 2007, Araújo et al. 2011). While, Guapira opposita (Vell.) Reitz, and Davilla rugosa Poir. were reported in Restinga (Maia \& Oliveira 2010) and Amazon rainforest (Almada \& Fernandes 2011) areas, respectively. These reports demonstrate the importance of vegetation in the diversity of galls.
Galls were found predominantly on leaves in the present study, corroborating a pattern commonly reported in the scientific literature. It is estimated that approximately $70 \%$ of the galls throughout South America occur on leaves, with $20 \%$ on stems and branches, $7 \%$ on floral buds, and only 1 to $2 \%$ on (mature) flowers, or fruits and roots (Gonçalves-Alvim \& Fernandes 2001). The greatest occurrence of galls on leaves corroborates the pattern observed in Cerrado sites (GonçalvesAlvim \& Fernandes 2001, Almada \& Fernandes 2011, Araújo et al. 2011, Maia 2011) as well as in other Brazilian phytophysiognomies (Maia \& Fernandes 2004, Santos et al. 2012, Maia 2013a). When 


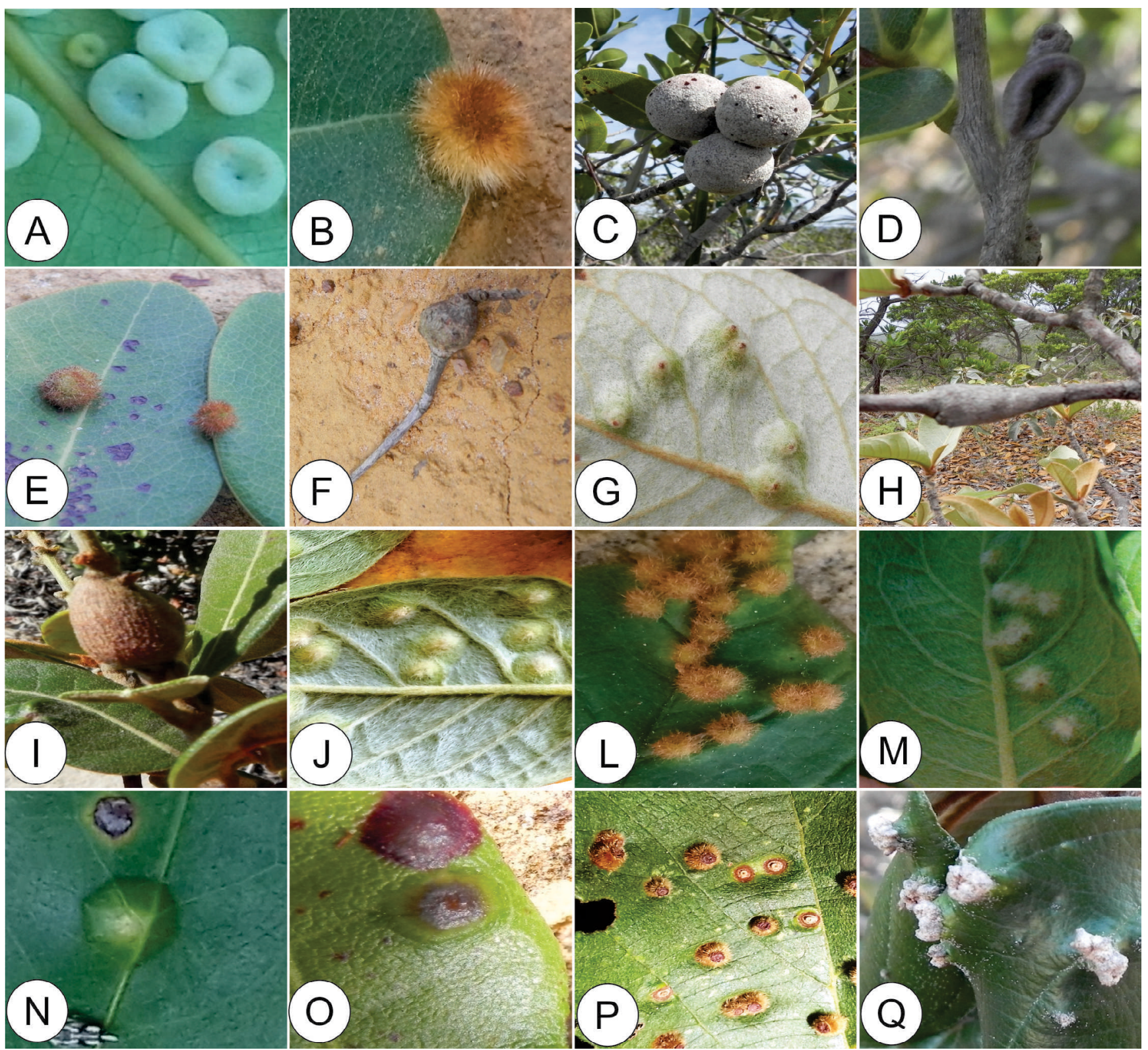

Figure 3. Insect galls in Rupestrian field and Cerrado stricto sensu vegetation in Caetité, BA, Brazil. A. Leaf gall on Copaifera langsdorffii (Leguminosae-Detarioideae). B-F. Galls on Copaifera sabulicola (Leguminosae-Detarioideae). B. Leaf gall. C. Stem gall. D. Bud gall. E. Leaf gall. F. Stem gall. G-H. Gall on Byrsonima cf. guilleminiana (Malpighiaceae). G. Leaf gall induced by Cecidomyiidae. H. Stem gall. I. Stem gall on Malpighiaceae Indet.1. J. Leaf gall on Malpighiaceae Indet. 2. L. Leaf gall on Malpighiaceae Indet. 3. induced by Cecidomyiidae. M. Leaf gall on Malpighiaceae Indet. 4. N. Leaf gall on Malpighiaceae Indet 5. O. Leaf gall on Malpighiaceae Indet. 6. P. Leaf gall on Luehea deviricata (Malvaceae). Q. Leaf gall on Miconia alborufescens (Melastomataceae).

considering the occurrence of galls on the available leaf surfaces, the abaxial surface was observed to hold $33 \%$ of the morphotypes, as it offers less stressful microclimatic conditions than the adaxial surface (Edward \& Wratten 1998).

The globoid, lenticular, and fusiform galls observed in the present study likewise predominate in other Brazilian ecosystems (Santos et al. 2010, Maia \& Souza 2013, Santos \& Ribeiro 2015), with the fusiform morphotype being more frequently encountered among stem galls (Gonçalves-Alvim \& Fernandes 2001). The globoid gall has been observed as the most common morphotype both in Cerrado and Rupestrian field sites (Araújo et al. 2007, Carneiro et al. 2009, Coelho et al. 2009, Nogueira et al. 2016).
Isolated and unilocular galls are most frequently cited in literature (Santos et al. 2011, Luz et al. 2012, Nogueira et al. 2016). This morphotype was frequently observed in dryland Caatinga areas in Pernambuco State (Santos et al. 2011), in northern Minas Gerais State (Luz et al. 2012), and in the Cerrado vegetation in Caetité (BA) (Nogueira et al. 2016). Presumably, isolated galls benefit those gall-inducing insects by better protecting them from natural enemies (Mani 1964).

Green-colored galls were only observed on leaves indicating that those structures probably produce photosynthates (Castro et al. 2012). A similar pattern was observed among galls examined by Araújo et al. (2007) and Costa et al. (2014b). Some galls have been observed to 


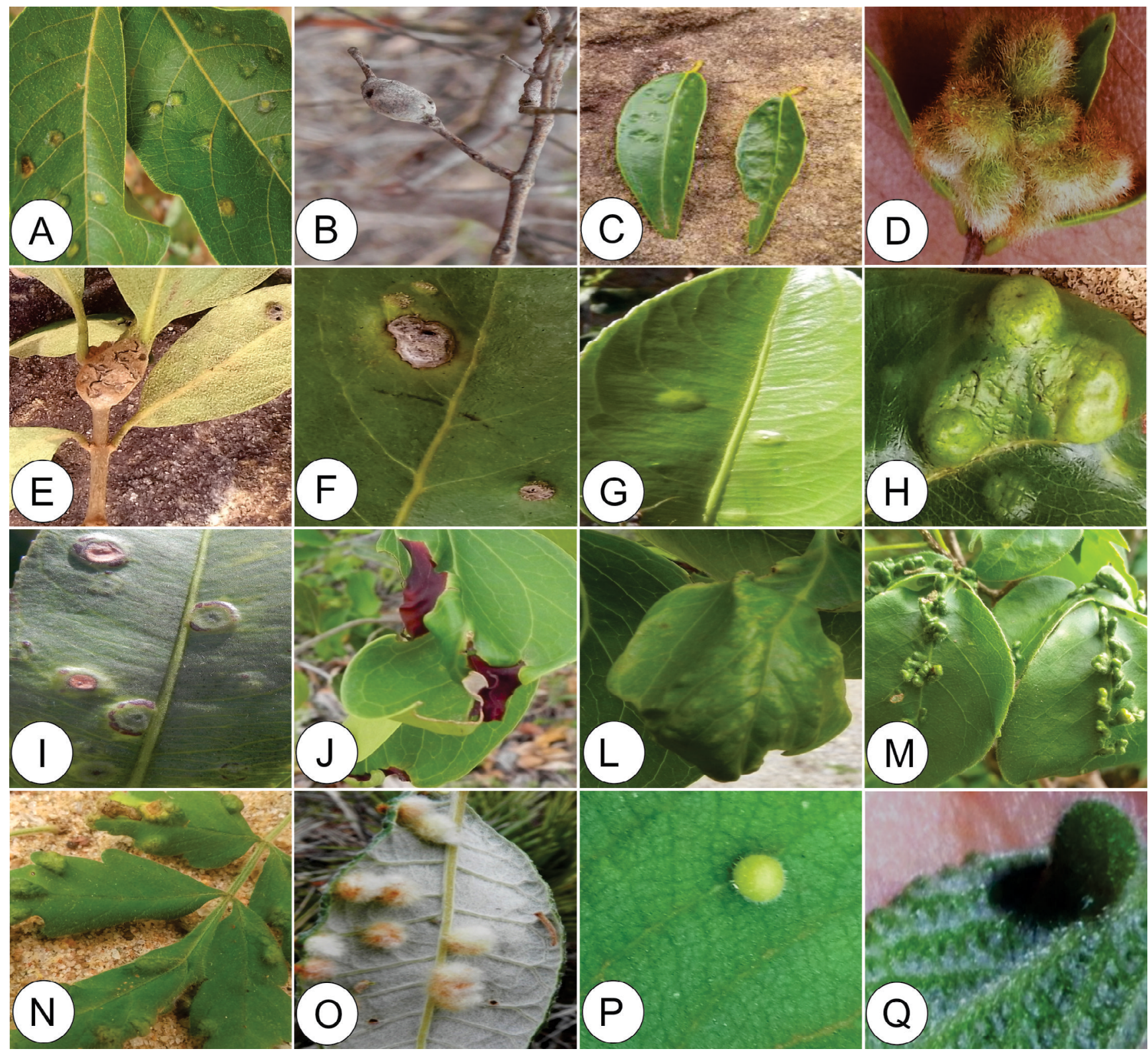

Figure 4. Insect galls in Rupestrian field and Cerrado stricto sensu vegetation in Caetité, BA, Brazil. A. Leaf gall on Campomanesia sp. 1 (Myrtaceae). B. Stem gall on Eugenia punicifolia (Myrtaceae). C. Leaf gall on Eugenia sp. 1. D. Leaf gall induced by Cecidomyiidae on Myrciaria cuspidata (Myrtaceae). E-F. Galls on Guapira opposita (Nyctaginaceae). E. Stem gall. F. Leaf gall induced by Diptera. G-I. Galls on Ouratea sp. 1 (Ochnaceae). G. Leaf gall induced by Cecidomyiidae. H. Leaf gall. I. Leaf gall. J-L. Galls on Roupala montana (Proteaceae). J. Marginal roll gall. L. Leaf gall. M. Leaf gall on Galipea sp. (Rutaceae). N. Leaf gall on Serjania paludosa (Sapidaceae). O. Leaf gall induced by Cecidomyiidae on Trigonia nivea (Trigoniaceae). P. Leaf gall induced by Cecidomyiidae on Lippia cf. alba (Verbenaceae). Q. Leaf gall on Lippia microphylla.

change color during their growth. Although those alterations are not well understood, they are probably associated with the growth of the galling insects, the developmental phase of the gall itself or the action of other trophic levels (Dias et al. 2013).

Our observations of pubescence on galls corroborated reports by other investigators (Araújo et al. 2007, Santos et al. 2010, Costa et al., 2014b, Nogueira et al. 2016), and showed that most of the morphotypes observed were glabrous. When present, however, trichomes can protect the gall-inducing insects from predators and parasites (Fernandes et al. 2009). Additionally, the presence of trichomes on the galls will help preventing water losses and favor temperature regulation within the structure (Stone \& Schonrögge 2003).
Our results corroborated studies that indicated Cecidomyiidae as the largest and most diverse group of gall-inducing insects in the Neotropical region (Gagné \& Jaschhof 2017). Studies in different Brazilian ecosystems such as Cerrado, Caatinga, Rupestrian field, and Restinga have also reported the predominance of this insect family (e.g., Maia 2001, Maia 2005, Carneiro et al. 2009, Santos et al. 2010). The Cecidomyiidae is currently known to comprise 6,590 species distributed among 812 genera (Gagné \& Jaschhof 2017). The Hymenoptera have been identified as the principal natural enemies of gall-inducing insects, commonly acting as parasitoids, the principal cause of mortality among the first; their habits were verified in the present study as well as in other 
Galls in Rupestrian field and Cerrado

Table 2. Richness of gall morphotypes in host plants of Cerrado and Rupestrian field in Brazil

\begin{tabular}{|c|c|c|c|c|c|c|}
\hline Locality & Biomes & Richest families & $\begin{array}{c}\text { Number of } \\
\text { morphotypes }\end{array}$ & $\begin{array}{c}\text { Mean of } \\
\text { morphotypes }\end{array}$ & Richest genera & Richest species \\
\hline $\begin{array}{l}\text { Brejinho das } \\
\text { ametistas, Caetité, } \\
\text { BA (the present } \\
\text { study) }\end{array}$ & $\begin{array}{l}\text { Rupestrian fields } \\
\text { Cerrado }\end{array}$ & $\begin{array}{l}\text { Leguminosae (10), } \\
\text { Malpighiaceae ( } 8) \\
\text { e Myrtaceae (4) }\end{array}$ & 48 & 1.3 & $\begin{array}{l}\text { Copaifera } \text { L. (5) } \\
\text { Mimosa L. (2) } \\
\text { Byrsonima Rich. ex } \\
\text { Kunth (2) }\end{array}$ & Copaifera langsdorffii Desf.(5) \\
\hline $\begin{array}{l}\text { Serra de Caetité, } \\
\text { BA (Nogueira et al. } \\
\text { 2016) }\end{array}$ & Cerrado-Caatinga & $\begin{array}{l}\text { Leguminosae (22) } \\
\text { Malpighiaceae (10) }\end{array}$ & 49 & 3.5 & $\begin{array}{l}\text { Copaifera }(10) \\
\text { Bauhinia } \text { L. }(6) \\
\text { Mimosa }(4)\end{array}$ & $\begin{array}{l}\text { Copaifera langsdorffii Desf.(9) } \\
\text { Bauhinia acuruana Moric. (5) }\end{array}$ \\
\hline $\begin{array}{l}\text { Serra do Cabral, } \\
\text { MG (Coelho et al. } \\
\text { 2013b) }\end{array}$ & Cerrado & $\begin{array}{l}\text { Asteraceae (12) } \\
\text { Malpighiaceae (9) } \\
\text { Fabaceae (4) }\end{array}$ & 47 & 1.2 & $\begin{array}{l}\text { Byrsonima }(6) \\
\text { Lessingianthus } \mathrm{H} . \\
\text { Rob. (4) }\end{array}$ & $\begin{array}{l}\text { Byrsonima guilleminiana A. Juss. } \\
\text { (3) }\end{array}$ \\
\hline $\begin{array}{l}\text { Cadeia do } \\
\text { Espinhaço, MG } \\
\text { (Carneiro et al. } \\
\text { 2009) }\end{array}$ & Rupestrian fields & $\begin{array}{l}\text { Asteraceae (47) } \\
\text { Melastomataceae } \\
(26) \\
\text { Malpighiaceae (22) } \\
\text { Leguminosae (21) }\end{array}$ & 241 & 1.7 & $\begin{array}{l}\text { Baccharis L. (39) } \\
\text { Byrsonima (17) } \\
\text { Microlicia D.Don } \\
\text { (10) } \\
\text { Lessingianthus } \\
\text { Lychnophora Mart. } \\
(9)\end{array}$ & $\begin{array}{l}\text { Baccharis pseudomyriocephala } \\
\text { Teodoro Luis (10) } \\
\text { Byrsonima coccolobifolia Kunth (8) }\end{array}$ \\
\hline $\begin{array}{l}\text { Serra do Cipó, MG } \\
\text { (Coelho et al. 2009) }\end{array}$ & Rupestrian fields & $\begin{array}{l}\text { Leguminosae (22) } \\
\text { Myrtaceae (11) } \\
\text { Asteraceae (8) }\end{array}$ & 92 & 1.8 & $\begin{array}{l}\text { Bauhinia (10) } \\
\text { Myrcia (7) } \\
\text { Baccharis (6) } \\
\text { Celtis L. (6) } \\
\text { Serjania Mill. (6) }\end{array}$ & $\begin{array}{l}\text { Baccharis dracunculifolia DC. (5) } \\
\text { Celtis brasiliensis (Gardner) } \\
\text { Planch. (5) }\end{array}$ \\
\hline
\end{tabular}

inventories (Maia 2001, Maia \& Fernandes 2004, Maia et al. 2008, Maia \& Azevedo 2009).

The plants encountered in the Cerrado demonstrated greater richness of galls than those in Rupestrian field vegetation, although the latter phytophysiognomy is undoubtedly important in terms of the diversity of entomogenous galls in Brazil. As such, more surveys will be necessary to cover areas not yet examined and complement our knowledge on the richness and morphology of galls. The biology of gall-inducing insects, the relationships between those insects and their host plants, and the dynamics the guild of gall-inducing insects in Rupestrian field vegetation, should respond to the environmental variations and indicate the quality of the habitat.

\section{Acknowledgments}

This work was supported by the Departamento de Ciências Humanas da Universidade do Estado da Bahia, Campus VI, by Programa de Pós-Graduação em Biodiversidade Vegetal (PPGBveg/UNEB), by the Conselho Nacional de Desenvolvimento Científico e Tecnológico (Proc. no 406111/2016-2), and by the Fundação de Amparo à Pesquisa do Estado da Bahia (Proc. no 9648/2015). The authors thank FAPERJ for the study grant awarded to S.P. Carvalho-Fernandes (Proc. E-26/203.170/2015), the researchers of the Universidade Estadual de Feira de Santana (UEFS) for their help in identifying the botanical material, and Marcelo Vasconcelos for providing a map of the study area.

\section{Author contributions}

Leir Guimarães Vieira: Contribution to data collection;

Ravena Malheiros Nogueira: Contribution to manuscript preparation;

Elaine Cotrim Costa: Contribution to data analysis and interpretation;

Sheila Patrícia Carvalho-Fernandes: Contribution to data analysis and interpretation and to manuscript preparation;

Juliana Santos Silva: Substantial contribution in the concept and design of the study.

\section{Conflicts of interest}

The authors declare that they have no conflict of interest related to the publication of this manuscript. 


\section{References}

ALMADA, E.D. \& FERNANDES, G.W.A. 2011. Insetos indutores de galhas em florestas de terra firme e em reflorestamentos com espécies nativas na Amazônia Oriental, Pará, Brasil. Bol. Mus. Para. Emílio Goeldi. Cienc. Nat. 6(2): 163-196.

APG IV. 2016. An update of the Angiosperm Phylogeny Group classification for the orders and families of flowering plants: APG IV. Bot. J. Linn. Soc. 181: $1-20$.

ARAÚJO, W.S., KLEIN. V.L.L.G. \& SANTOS. B.B. 2007. Galhas Entomógenas Associadas à Vegetação do Parque Estadual da Serra dos Pireneus, Pirenópolis, Goiás, Brasil. R. Bras. Bioci. 5: 45-47.

ARAÚJO, W.S., SANTOS B.B. \& GOMES-KLEIN, V.L. 2011. Insect galls from Serra dos Pireneus, GO, Brazil. Biota Neotrop. 11 (2): 357-365.

ARAÚJO, W.S., PORFÍRIO-JÚNIOR, E.D., JORGE, V.A. \& FILHO, K.S. 2012. Plantas hospedeiras e galhas entomógenas em sub-bosques de florestas Tropicais do Pará, Brasil. INSULA 41: 59-72.

ARAÚJO, W.S., SOBRAL, F.L. \& MARACAHIPES, L., 2014. Insect galls of the Parque Nacional das Emas (Mineiros, GO, Brazil). Check List 10 (6): 1445-1451.

CARNEIRO, M.A.A., BORGES, R.A.X., ARAÚJO, A.P.A. \& FERNANDES, G.W. 2009. Insetos indutores de galhas da porção sul da Cadeia do Espinhaço, Minas Gerais, Brasil. Rev. Bras. Entomol. 53(4): 570-592.

CARVALHO-FERNANDES, S.P., ALMEIDA-CORTEZ, J.S. \& FERREIRA, A.L.N. 2012. Riqueza de galhas entomógenas em áreas antropizadas e preservadas de Caatinga. Rev. Árvore 36 (2): 269-277.

CASTRO, A.C.R., LEITE, G.L.D., OLIVEIRA, D.C.\& ISAIAS, R.M.S. 2012. Morphological Patterns of a Hymenopteran Gall on the Leaflets of Caryocar brasiliense Camb. (Caryocaraceae). Am. J. Plant Sci. 3 (7): 921-929.

CEI - Centro de Estatística e Informações. 1994. Informações básicas de municípios baianos: Região Serra Geral. Salvador, Bahia.

COELHO, M.S., ALMADA, E.D., FERNANDES, G.W., CARNEIRO, M.A.A., SANTOS, M.S., QUINTINO, A.V. \& SANCHEZ-AZOFEIFA, A. 2009. Gall inducing arthropods from a seasonally dry tropical forest in Serra do Cipó, Brazil. Rev. Bras. Entomol. 53 (3): 404-414.

COELHO, M.S., CARNEIRO, M.A.A., BRANCO, C., BORGES, R.A.X. \& FERNANDES, G.W. 2013a. Gall-inducing insects from Campos de Altitude, Brazil. Biota Neotrop. 13(4): 139-151.

COELHO, M.S., CARNEIRO, M.A.A., BRANCO, C. \& FERNANDES, G.W. 2013b. Insetos indutores de galhas da Serra do Cabral, Minas Gerais, Brasil. Biota Neotrop. 13(3):102-109.

COSTA, F.V., FAGUNDES, M., NEVES, F.S., 2010. Arquitetura da planta e diversidade de galhas associadas à Copaifera langsdorffii (Fabaceae). Ecol. Austral. 20, 9-17.

COSTA, E.C., CARVALHO-FERNADES, S.P. \& SANTOS-SILVA, J., 2014a. Galhas entomógenas associadas à Leguminosae do entorno do riacho Jatobá, Caetité, Bahia. Brasil. Rev. Bras. Bioci. 12 (2): 115-120.

COSTA, E.C., CARVALHO-FERNANDES, S.P. \& SANTOS-SILVA, J. 2014b. Galhas de insetos em uma área de transição caatinga-cerrado no Nordeste do Brasil. Sitientibus Sér. Ciên. Biol. 14, 1-9.

COSTA, E.C. 2016. Diversidade de Galhas de insetos em áreas de Cerrado no Parque Estadual da Serra dos Montes Altos, Bahia. Dissertação (Mestrado), Universidade do Estado da Bahia, Departamento Educação, Paulo Afonso, BA.

DIAS, G.G., FERREIRA, B.G., MOREIRA, G.R.P. \& E ISAIAS, R.M.S. 2013. Developmental pathway from leaves to galls induced by a sap-feeding insect on Schinus polygamus (Cav.) Cabrera (Anacardiaceae). An. Acad. Bras. Cienc. 85(1): 187-200.

EDWARD, P. J. \& WRATTEN, S.D. 1998. Ecology of insect-plant interations. Southampton, The Camelot Press. 60p.

ESPIRITO-SANTO, M.M. \& FERNANDES, G.W. 2007. How Many Species of Gall-Inducing Insects Are There on Earth, and Where Are They? Ann. Entomol. Soc. Am. 100 (2): 95-99.
FERNANDES, S.P.C., CASTELO-BRANCO, B.P., ALBUQUERQUE, F.A., FERREIRA, A.L.N., BRITO-RAMOS, A.B., BRAGA, D.V. \& ALMEIDACORTEZ, J. 2009. Galhas entomógenas em um fragmento urbano de Mata Atlântica no centro de endemismo de Pernambuco. Rev. Bras. Bioci. 7 (3): 240-244.

FLECK, T. \& FONSECA, C.R. 2007. Hipóteses sobre a riqueza de insetos galhadores: uma revisão considerando os níveis intra-específico, interespecífico e de comunidade. Neot. Biol. Conserv. 2(1):36-45.

FORMIGA, A.T., SOARES, G.L.G. \& ISAIAS, R.M. 2011. Responses of the Host Plant Tissues to Gall Induction in Aspidosperma spruceanum Müell. Arg. (Apocynaceae). Am. J. Plant Sci 2: 823-834.

GAGNÉ, R.J. \& JASCHHOF, M. 2017. A Catalog of the Cecidomyiidae (Diptera) of the World. Fourth Edition. Digital. 762 pp.

GONÇALVES-ALVIM, S.J. \& FERNANDES, G.W. 2001. Comunidades de insetos galhadores (Insecta) em diferentes fisionomias do Cerrado em Minas Gerais, Brasil. Rev. Bras. Zool. 18: 289-305.

ISAIAS, R.M.S., CARNEIRO, R.G.S., OLIVEIRA, D.C. \& SANTOS, J.C. 2013. Illustrated and Annotated Checklist of Brazilian Gall Morphotypes. Neotrop. Entom. 42: 230-239.

LUZ, G.R., FERNANDES G.W. \& SILVA, J.O., 2012. Galhas de insetos em habitats xérico e mésico em região de transição Cerrado-Caatinga no norte de Minas Gerais, Brasil. Neotrop. Biol. Conserv. 7: 171-187.

MAIA, V.C. 2001. The gall midgs (Diptera, Cecidomyiidae) from three restingas of Rio de Janeiro State, Brazil). Rev. Brasil. Zool. 18:583-629.

MAIA, V.C. \& FERNANDES, G.W. 2004. Insect galls from Serra de São José (Tiradentes, MG, Brazil). Braz. J. Biol. 64(3): 423-445.

MAIA, V.C. 2005. Clinodiplosis costai, a new galler species (Diptera, Cecidomyiidae) associated with Paullinia weinmanniaefolia Mart. (Sapindaceae). Rev. Bras. Zool. 22 (3): 676-679.

MAIA. V.C, MARGENTINA. M.A.G. \& MARTINS, E.S. 2008. Ocorrência e caracterização de galhas de insetos em áreas de restinga de Bertioga (São Paulo, Brasil). Biota Neotrop. 8 (1):167-197.

MAIA, V.C. \& AZEVEDO, M.A.P. 2009. Micro-himenópteros associados com galhas de Cecidomyiidae (Diptera) em Restingas do Estado do Rio de Janeiro (Brasil). Biota Neotrop. 9(2): 1-14.

MAIA, V.C. \& OLIVEIRA, J.C. 2010. Galhas de insetos da Reserva Biológica Estadual da Praia do Sul (Ilha Grande, Angra dos Reis, RJ). Biota Neotrop. 10 (4): 227-237.

MAIA, V.C. 2011. Galhas de insetos do Parque Nacional do Ibitipoca (Minas Gerais, Brasil). X Congresso de Ecologia do Brasil. Minas Gerais, 2009.

MAIA, V.C. 2013a. Insect galls of São Tomé das Letras (MG, Brazil). Biota Neotrop. 13 (4): 164-189.

MAIA, V.C. 2013b. Galhas de insetos em restingas da região sudeste do Brasil com novos registros. Biota Neotrop. 13(1): 183-209.

MAIA, V.C. \& SOUZA, M.C. 2013. Insect galls of the xeric vegetation of Ilha do Cabo Frio (Arraial do Cabo, RJ, Brazil). Biota Neotrop. 13 (3):278-288.

MINISTÉRIO DO MEIO AMBIENTE - MMA. 2007. Áreas Prioritárias para Conservação, Uso Sustentável e Repartição de Benefícios da Biodiversidade Brasileira: Atualização - Portaria MMA n ${ }^{\circ}$ 9, de 23 de janeiro de 2007, Secretaria de Biodiversidade e Florestas. - Brasília: MMA, 2007. 300 pp.

NOGUEIRA, R.M., COSTA, E.C. \& SANTOS-SILVA, J. 2016. Levantamento de Galhas entomógenas na Serra Geral, Caetité, Bahia, Brasil. Biota Neotrop. 16(1): e20150035.

OLIVEIRA, J.C. \& MAIA, V.C. 2005. Ocorrência e caracterização de galhas de insetos na restinga de Grumari (Rio de Janeiro, RJ, Brasil). Arch. Mus. Nac. (Rio de J.) 63: 669-675.

OLIVEIRA, D.C., DRUMMOND, M.M., MOREIRA, A.S.F.P., SOARES, G.L.G. \& ISAIAS, R.M.S. 2008. Potencialidades morfogênicas de Copaifera langsdorffii Desf. (Fabaceae): super-hospedeira de herbívoros galhadores. Rev. Biol. Neotrop. 5(1): 31-39.

OLIVEIRA, D.C., MENDONÇA JR., M.S., MOREIRA, A.S.F.P., LEMOSFILHO, J.P. \& ISAIAS, R.M.S. Water stress and phenological synchronism between Copaifera langsdorffii (Fabaceae) and multiple galling insects: formation of seasonal patterns. 2014. J Plant Interact 8(3): 225-233. 
PRICE, P.W., FERNANDES, G.W., WARING, G.L., 1987. Adaptive Nature of Insect Galls. Environ. Entomol. 16: 15-24.

SANTOS, J.C., ALMEIDA-CORTEZ, J.S. \& FERNANDES, G.W. 2009. Diversity of gall-inducing insects in the high altitude wetland forests in Pernambuco, Northeastern Brazil. Braz. J. Biol. 71: 47-56.

SANTOS, B.B., FERREIRA, H.D. \& ARAÚJO, W.S. 2010. Ocorrência e caracterização de galhas entomógenas em uma área de floresta estacional semidecídua em Goiânia, Goiás, Brasil. Acta Bot. Bras. 24: 243-249.

SANTOS, J.C., ALMEIDA-CORTEZ, J.S. \& FERNANDES, G.W. 2011. Richness of gall-inducing insects in the tropical dry forest (Caatinga) of Pernambuco. Rev. Bras. Entomol. 55: 45-54.

SANTOS, J.C., ALMEIDA-CORTEZ, J.S. \& FERNANDES, G.W. 2012.Gallinducing insects from Atlantic forest of Pernambuco, Northeastern Brazil. Biota neotrop. 12: 197-213.

SANTOS, P. O \& RIBEIRO, J.E.L.S. 2015. Ocorrência e caracterização de galhas em fragmento de Floresta Estacional Semidecidual em Telêmaco Borba, Paraná, Brasil. Semina 36 (2): 15-24.

SILVA, M.H.M., COSTA-FILHO, L.O., OLIVEIRA, A.F.M. \& ALMEIDACORTEZ, J.S. 2011. Ocorrência de Galhas em Espécies de Croton do Parque Nacional Vale do Catimbau (PE). Rev. Bras. Geo. Fís. 3: 510-519.
STONE, G.N. \& SCHÖNROGGE, K. 2003. The adaptive significance of insect gall morphology. Trends Ecol. Evol. 18: 512-522.

TABARELLI, M. 1998. Dois Irmãos: o desafio da conservação biológica em um fragmento de Floresta Tropical. In Reserva Ecológica de Dois Irmãos: estudos em um remanescente de Mata Atlântica em área urbana (RecifePernambuco-Brasil) (I.C. Machado, A.V. Lopez \& K.C. Porto, eds). Ed. Universitária, Universidade Federal de Pernambuco, p. 311-323.

URSO-GUIMARÃES, M.V. \& SCARELI-SANTOS, C. 2006. Galls and gall makers in plants from the Péde-gigante Cerrado Reserve Santa Rita do Passa Quatro, SP, Brazil. Braz. J. Biol. 66: 357-369.

URSO-GUIMARÃES, M.V., CASTELLO, A.C.D., KATAOKAA, E.Y. \& KOCHC, I. 2017. Characterization of entomogen galls from Mato Grosso do Sul. Brazil. Rev. Bras. Entomol. 61: 25-42.

VASCONCELOS, M.F., SOUZA, F.J., PARRINI, R., SERPA, G.A., ALBANO, C., ABREU, C.R.M., SANTOS. S.S. \& NETO, F.P.F. 2012. The avifauna of Brejinho das Ametistas, Bahia, Brazil: birds in a caatinga-cerrado transitional zone, with comments on taxonomy and biogeography. Rev. Bras. Orn. 20 (3): 246-267.

VELDTMAN, R. \& MCGEOCH, M.A. 2003. Galling-insect species richness along a non-scleromorphic vegetation gradient in South Africa: a test of the hygrothermal stress hypothesis. Aust. J. Ecol. 28: 1-13. 